\title{
Prestation de soins de maternels respectueux: conclusions d'une evaluation à petite echelle à Chipata en Zambie
}

Breakthrough RESEARCH

Follow this and additional works at: https://knowledgecommons.popcouncil.org/departments_sbsr-rh How does access to this work benefit you? Let us know!

\section{Recommended Citation}

Breakthrough RESEARCH. 2020. "Prestation de soins de maternels respectueux : conclusions d'une évaluation à petite échelle à Chipata en Zambie, » Dossier de recherche programmatique. Washington, DC: Population Council. 
I'USAID et I'Office sanitaire du district (OSD) de Chipata en Zambie. Les conclusions de la phase de recherche formative ont contribué au développement de solutions par le biais d'un processus de modélisation itératif axé sur les comportements. Ce processus a abouti au développement d'un ensemble de solutions articulé autour de cinq composantes : (1) Boîte à outils BETTER pour la prise en charge de la douleur, (2) boîte à retours, (3) promesse prestataire-cliente, (4) fonds Fresh Start, et (5) atelier de réflexion. De plus amples détails sur les modèles figurent dans l'encadré 1. Ce dossier décrit les principales conclusions et les points à retenir concernant l'effet des solutions sur l'expérience des clientes en matière de soins au cours de leur phase de travail et de leur accouchement.

La théorie du changement (Figure 1) décrit notre compréhension des trajectoires des SMR à travers le changement de comportement des prestataires et présente notre conceptualisation de la manière dont nos solutions influenceront le comportement des agents de santé, en nous focalisant sur I'individu à l'origine de ce préjudice ou mauvais traitement.

\section{Modèle de recherche}

Nous avons réalisé un test pilote de l'ensemble de solutions SMR dans 10 établissements urbains et ruraux à Chipata en Zambie. Les structures pouvaient être incluses si SM360+ y opéraient certains programmes. Les cinq composantes de l'ensemble de solutions ont été introduites dans cinq structures et correspondaient à cinq établissements témoins sur la base des critères suivants : nombre moyen d'accouchements mensuels, nombre d'agents du personnel, taille de la population dans la zone de desserte et distance les séparant de I'OSD (centre-ville).

En septembre 2019, nous avons recueilli des données de base au sein des structures d'intervention et des établissements témoins avant de procéder à une collecte de données finales en décembre de la même année. Les enquêtes auprès des prestataires nous ont permis de recueillir leurs perceptions par rapport aux SMR, y compris l'expérience des patientes et les niveaux de relationnel, l'empathie et la confiance qui existaient entre les prestataires et les clientes. Les clientes ont été interrogées quatre à huit semaines après leur accouchement, selon les recommandations de la littérature SMR, afin de saisir le plus précisément possible l'expérience

\section{ENCADRÉ 1 SOLUTIONS SMR}

\section{Vous trouverez ci-dessous une liste complète des cinq composantes de l'ensemble de solutions SMR. Pour en savoir plus sur les solutions et les obstacles comportementaux auxquels elles tentent de remédier, veuillez consulter notre dossier programmatique précédent et le manuscrit ici.}

Boîte à outils BETTER pour la prise en charge de la douleur : BETTER est un acronyme anglais signifiant « Meilleur », formé à partir des mots « Breathe » (respirer), « Encourage » (encourager), « Turn » (se retourner), « Think » (penser) et « Rub » (masser). Cette boîte à outils a pour but d'incorporer la gestion de la douleur dans les soins de routine dispensés aux clientes. Elle comprend : (1) les affiches BETTER sur la technique de soulagement de la douleur, (2) le manuel BETTER sur la prise en charge de la douleur, (3) des balles de massage et (4) le guide BETTER du partogramme pour le soulagement de la douleur.

Boîte à retours : à leur sortie de la maternité, les femmes recevaient un jeton qu'elles devaient insérer dans l'une des fentes de la boîte reflétant le mieux leur niveau de satisfaction par rapport au service dispensé. La boîte à retours avait pour but d'amener les clientes à faire part de leurs commentaires et impressions concernant les services reçus.

Promesse prestataire-cliente : la promesse avait pour objectif de clarifier et de fixer certaines attentes en matière de comportement, aussi bien pour les prestataires que pour les clientes et de rassurer les patientes quant au traitement qu'elles devaient recevoir. Le document était lu à voix haute par les prestataires au moment de l'admission des patientes à la maternité et était signé par le prestataire et la cliente.

Fonds Fresh Start : les établissements de santé ont reçu un financement restreint qu'elles ont utilisé pour procéder à des changements dans la salle de travail, afin d'améliorer l'expérience non cliniques des patientes. Le fonds Fresh Start visent à générer un sentiment de nouveau départ pour le personnel en lui donnant le pouvoir de changer l'expérience des clientes en matière de soins.

Atelier de réflexion : I'atelier a encouragé/ les prestataires à livrer une réflexion sur les soins dispensés, à formuler l'intention de changer la manière d'administrer les soins au sein de la structure et à introduire diverses solutions. 


\section{FIGURE 1 THÉORIE DU CHANGEMENT}

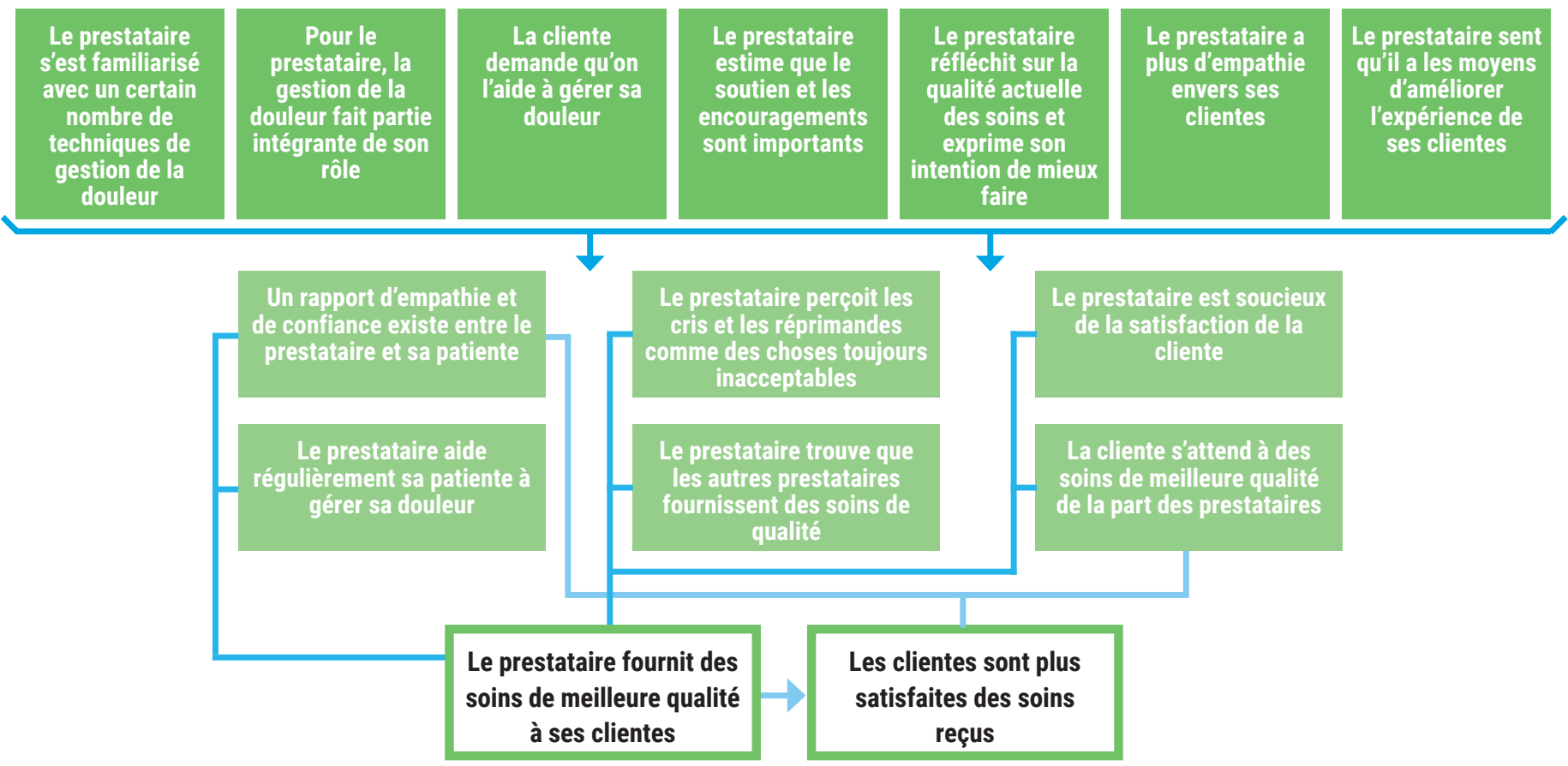

liées au manque de respect et aux cas d'abus durant l'accouchement. ${ }^{3}$ On a également demandé aux prestataires et aux clientes de répondre à des questions qualitatives ouvertes au cours de l'étude finale quant à leurs perceptions des soins et de l'ensemble de solutions. Nous avons également procédé à des visites de suivi et des entretiens auprès des responsables de structures au sein des établissements de mise en œuvre au fil de la période d'exécution du programme. Les tableaux 1 et 2 donnent un aperçu des participants à l'enquête.

Nous avons évalué l'efficacité de l'ensemble de solutions SMR sur les résultats décrits dans la théorie du changement pour déterminer si les interventions avaient amélioré l'expérience des clientes en matière de soins durant leur accouchement au sein de structures de santé. Nous avons mesuré les différences dans les résultats entre les groupes d'intervention et témoins et contrôlé certaines variables au cours de la phase finale. ${ }^{b}$ Dans la mesure où nous n'avons pas été en mesure d'affecter de manière aléatoire les établissements dans les groupes d'intervention ou témoins, une analyse de sensibilité supplémentaire a été réalisée pour valider les résultats. Dans les cas où les résultats de l'analyse de sensibilité ne validaient pas les observations, nous pensons qu'il existait une différence significative dans le résultat obtenu entre les groupes témoins et les groupes d'intervention, bien que la taille de

\footnotetext{
${ }^{b}$ Dans l'analyse des prestataires, nous avons contrôlé la variable de résultat au cours de l'étude de base, si un prestataire était une sage-femme, selon la dimension de genre, le nombre d'années d'expérience en matière d'accouchement et le nombre d'accouchements réalisés au cours des deux dernières semaines. Les contrôles de l'analyse au niveau des clientes comprenaient des moyennes de l'étude de base au niveau des structures pour chaque résultat à l'étude, l'état civil des clientes, leur âge et leur parité.
}

l'effet puisse être moins connue en raison des facteurs que nous ne pouvions pas contrôler. Des données qualitatives ont

\section{TABLEAU 1 DONNÉES DÉMOGRAPHIQUES DES PRESTATAIRES ENQUÊTÉS}

\begin{tabular}{|c|c|c|}
\hline Prestataires & $\begin{array}{l}\text { Étude de base } \\
\qquad(n=33)\end{array}$ & $\begin{array}{l}\text { Étude finale } \\
\qquad(n=35)\end{array}$ \\
\hline Sexe & $85 \%$ de femmes & $77 \%$ de femmes \\
\hline Catégorie & $\begin{array}{l}55 \% \text { de sages- } \\
\text { femmes }\end{array}$ & $\begin{array}{l}54 \% \text { de sages- } \\
\text { femmes }\end{array}$ \\
\hline $\begin{array}{l}\text { Nombre moyen d'années } \\
\text { d'expérience en matière d'ac- } \\
\text { couchement }\end{array}$ & 9,7 & 9,3 \\
\hline
\end{tabular}

\section{TABLEAU 2 DONNÉES DÉMOGRAPHIQUES DES CLIENTES ENQUÊTÉES}

\begin{tabular}{|lcc|}
\hline Clientes & $\begin{array}{c}\text { Étude de base } \\
(\mathbf{n}=60)\end{array}$ & $\begin{array}{c}\text { Étude finale } \\
(\mathbf{n}=92)\end{array}$ \\
\hline Âge & 23,5 ans & 24,5 ans \\
\hline État civil & $95 \%$ mariées & $79 \%$ mariées \\
\hline $\begin{array}{l}\text { Parité moyenne } \\
\begin{array}{l}\text { Temps écoulé en moyenne } \\
\text { depuis l'accouchement au } \\
\text { moment de l'enquête }\end{array}\end{array}$ & 2,5 enfants & 2,4 enfants \\
\hline
\end{tabular}


également été utilisées pour développer une compréhension plus nuancée des résultats. Les résultats décrits dans ce dossier sont statistiquement ou marginalement significatifs à a $p<0,10$.

\section{Conclusions principales}

Les conclusions présentées plus bas se rapportent principalement à l'efficacité du processus d'intervention et soulignent les enseignements supplémentaires tirés de ce dernier concernant les SMR et de manière plus générale, l'expérience des clientes.

\section{Manque de respect et violence}

\section{Le manque de respect et la violence vis-à-vis des clientes étaient moins fréquents.}

En fin d'intervention, selon leurs témoignages, les clientes prises en charge dans les établissements participants étaient beaucoup moins susceptibles (15\% de moins) d'avoir été victimes de manque de respect ou de violence par rapport aux clientes des établissements témoins. Les types de comportements irrespectueux et de violence signalés par les clientes en fin d'intervention étaient les suivants : manque d'intimité, menaces, accouchement seule, sentiment d'abandon et traitement mettant la patiente mal à l'aise. Les comportements irrespectueux étant plus rares dans les établissements participants, l'analyse de sensibilité n'a pas confirmé ces conclusions. En outre, l'échantillon étant limité, il est probable que des changements aussi minimes passent inaperçus dans les résultats.

Les prestataires n'ont noté aucune différence quant à la fréquence des actes irrespectueux et violents observés ou commis.

En fin d'intervention, les prestataires des établissements participants n'étaient pas plus susceptibles d'observer des comportements irrespectueux et violents que les prestataires des établissements témoins. Les prestataires, à la fois des établissements participants et témoins, ont indiqué avoir observé les quatre types de comportements irrespectueux et violents mis en évidence (recours à la force, menaces à l'encontre de la cliente, manque de respect à l'égard de la cliente en raison d'une stigmatisation sociale, et réprimandes), les réprimandes étant la forme d'irrespect la plus souvent rencontrée. Malgré la fréquence élevée d'observation de soins irrespectueux, les prestataires avaient tendance à déclarer que leurs collègues traitaient les clientes de manière acceptable. Un pourcentage bien inférieur de prestataires des établissements participants et témoins ont déclaré n'avoir rien fait de mal, et il n'y avait aucune différence notable entre les prestataires des établissements participants et témoins concernant les comportements irrespectueux et violents auto-signalés.

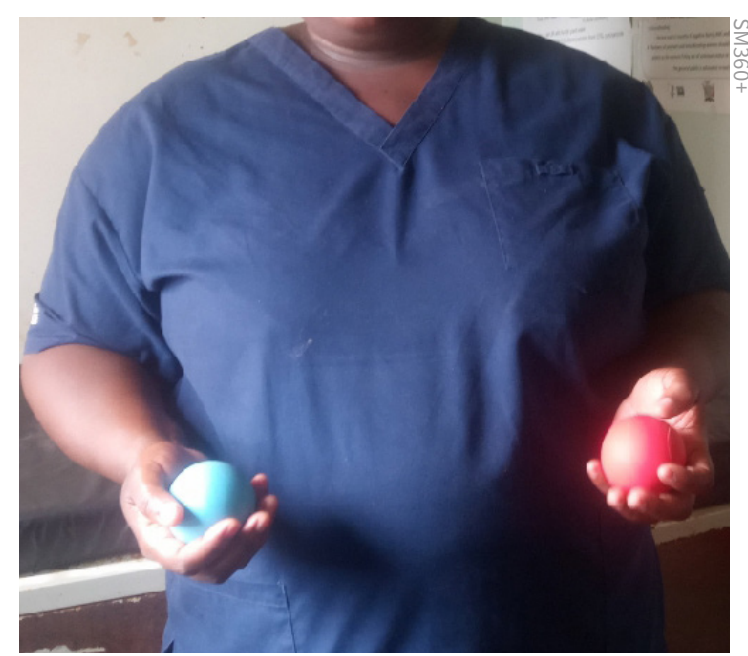

Les données recueillies ne nous permettent pas de savoir avec certitude si les prestataires ont intégré le fait que les cris ou les réprimandes étaient inadmissibles.

Selon des données peu significatives recueillies en fin d'intervention, en comparaison avec les prestataires des établissements témoins, les prestataires des établissements participants ont plus souvent déclaré que leurs confrères du même établissement estimaient que les cris ou les réprimandes à l'encontre d'une cliente étaient inadmissibles. L'analyse de sensibilité n'a pas confirmé ces conclusions. Les résultats suggèrent que même si certains prestataires ne cautionnent pas les cris, d'autres estiment qu'il est parfois justifié de hausser le ton dans certaines circonstances.

Le taux de surmenage des prestataires était faible et n'était pas lié à une implication auto-signalée dans les soins irrespectueux.

La section portant sur l'empathie et la déshumanisation du Maslach Burnout Inventory a été adaptée et appliquée. ${ }^{c}$ Les résultats du sondage ont révélé un taux de surmenage faible chez les prestataires en début d'intervention. Nous avons également découvert qu'il n'existait aucune corrélation entre le surmenage des prestataires et les cas de soins irrespectueux auto-signalés. Ces données confirment les conclusions tirées de nos recherches formatives, qui suggéraient que le surmenage ne constituait pas un facteur majeur de soins irrespectueux dans ce contexte.

\section{Gestion de la douleur}

\section{Les clientes étaient plus susceptibles de demander à ce qu'on} les aide à gérer la douleur.

Nous avons demandé aux clientes si elles avaient sollicité l'aide du prestataire lorsqu'elles ressentaient de la douleur.

\footnotetext{
'Le Maslach Burnout Inventory sert généralement à autoévaluer le niveau de surmenage. Nous avons modifié et adapté un certain nombre de ces questions pour les inclure dans notre sondage, notamment afin de mettre en évidence les cas de surmenage liés à la déshumanisation et à l'empathie.
} 
Les données recueillies suggèrent que les clientes prises en charge dans les établissements participants étaient beaucoup plus enclines à demander de l'aide pour gérer leur douleur que les clientes des établissements témoins : $77 \%$ des clientes dans les établissements participants contre $36 \%$ dans les établissements témoins. L'analyse de sensibilité a confirmé ces résultats.

Les prestataires avaient plus de chance de faire de la gestion de la douleur l'une de leurs priorités en tant que professionnels de santé.

Nous avons demandé aux prestataires de choisir les trois fonctions les plus importantes qu'ils devaient remplir pendant un accouchement, parmi une liste de tâches courantes retenues au cours de nos recherches formatives.

D’après les conclusions quasi significatives tirées de ces entretiens, en fin d'intervention, les prestataires des établissements participants avaient plus tendance à considérer la gestion de la douleur comme une priorité pendant l'accouchement par rapport aux prestataires des établissements témoins : $23 \%$ des prestataires des établissements participants contre $8 \%$ dans les établissements témoins. Même si l'analyse de sensibilité n'a pas confirmé ces résultats, nos données qualitatives suggèrent également que l'intervention a eu un impact significatif. Par exemple, les prestataires ont déclaré que les solutions de SMR les avaient sensibilisés à l'importance de leur rôle dans la gestion de la douleur de leurs clientes. Ils ont, en outre, souligné que la promesse prestataire-cliente leur avait également rappelé l'importance de soulager la douleur de leurs clientes.

Les techniques de gestion de la douleur employées par les prestataires ont évolué au fil du temps.

Nous avons constaté que les techniques employées par les prestataires des établissements participants n'étaient pas les mêmes en début et en fin d'intervention.

En début d'intervention, les techniques auxquelles les prestataires avaient le plus souvent recours lorsqu'une cliente demandait de l'aide étaient les massages, les encouragements et les conversations. En fin d'intervention, les trois techniques les plus couramment employées étaient les massages, les exercices de respiration et les changements de position, c'est-à-dire les techniques évoquées dans la Boîte à outils de gestion de la douleur BETTER.

De plus, plusieurs clientes ont déclaré que les balles de massage les avaient particulièrement soulagées et leur avaient procuré une expérience différente par rapport aux accouchements précédents.

J'ai apprécié la manière dont on m'a traitée, l'utilisation de la balle pour me masser le dos, la manière dont on m'a parlé lorsque j'avais mal et les encouragements qu'on m'a donnés.

-Témoignage d'une cliente ayant accouché dans un établissement participant

\section{FIGURE 2 TECHNIQUES DE GESTION DE LA DOULEUR EMPLOYÉES PAR LES PRESTATAIRES PARTICIPANTS PENDANT LA PHASE DE TRAVAIL ET L'ACCOUCHEMENT}
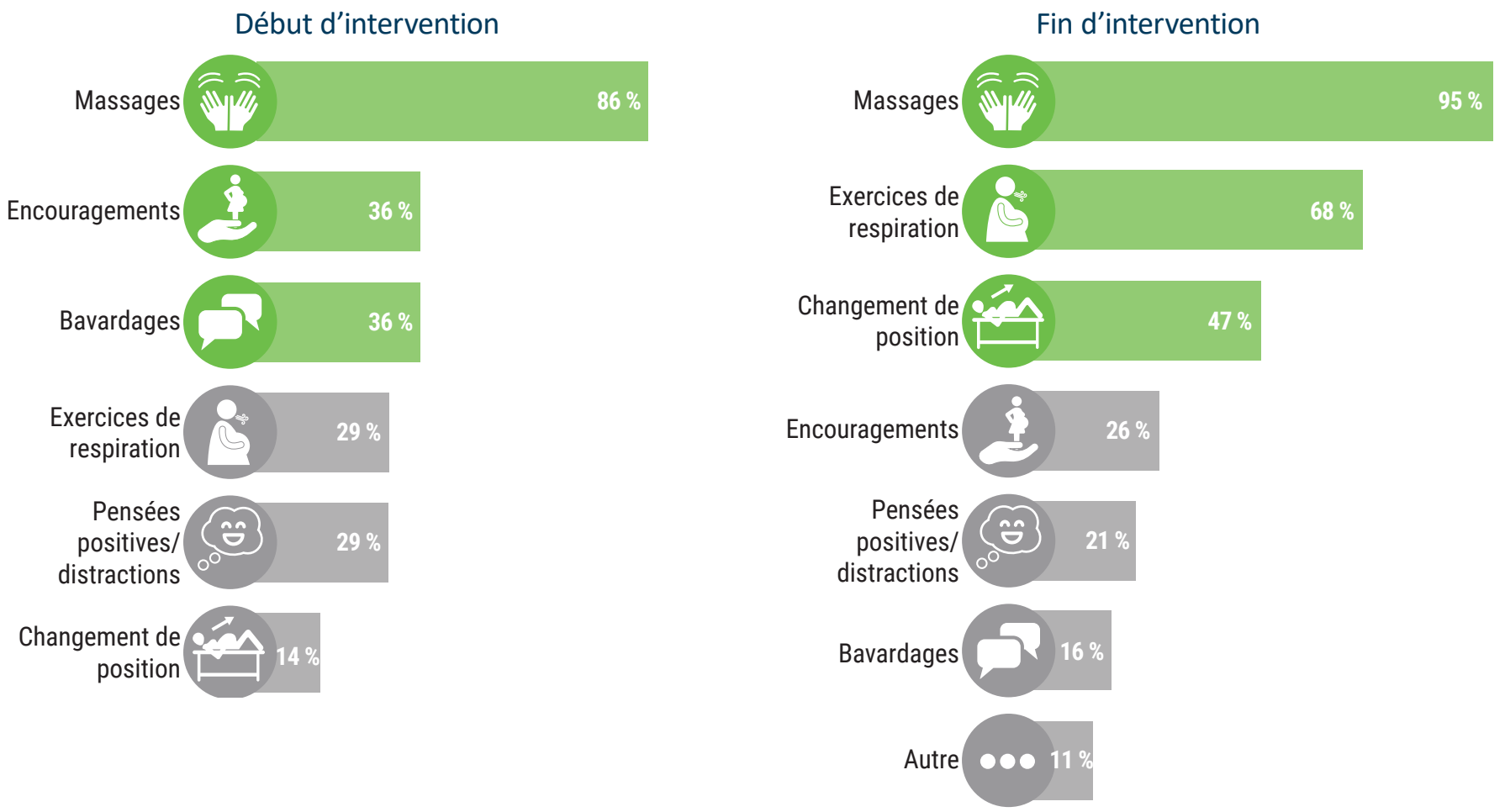


\section{Pouvoir d'améliorer la qualité des soins}

En début d'intervention, les prestataires étaient persuadés d'avoir les moyens d'améliorer la qualité des soins, et en fin d'intervention, cette impression n'avait pas changé.

Bien que l'ensemble des prestataires aient exprimé leur forte ou très forte volonté d'améliorer la qualité des soins, dans les établissements d'intervention et témoins, la plupart d'entre eux ont affirmé qu'aucune amélioration n'était nécessaire dans les établissements participants et témoins.

La plupart des prestataires des établissements à la fois participants et témoins ont affirmé que les soins prodigués au sein de leur établissement étaient de bonne qualité. Les prestataires ont vraisemblablement déjà confiance en leur capacité à faire évoluer les choses, et le fonds Fresh Start n'était pas nécessaires pour convaincre les prestataires qu'ils en avaient les moyens. D'autres solutions peuvent influencer le degré de confiance des prestataires, mais cela n'est pas leur but premier.

\section{Rapports de confiance et d'empathie entre le prestataire et la patiente}

Les prestataires étaient plus susceptibles d'adopter une attitude empathique à l'égard de leurs clientes et de reconnaitre que celles-ci sont coopératives.

Les résultats suggéraient qu'en fin d'intervention, les prestataires des établissements participants étaient nettement plus susceptibles d'être fortement d'accord avec le fait que les clientes sont souvent coopératives pendant le travail et l'accouchement, par rapport aux prestataires des établissements témoins. Les résultats sont également marginalement significatifs et suggèrent que les prestataires des établissements participants sont plus susceptibles d'être empathiques envers les clientes. L'analyse de sensibilité n'a cependant pas confirmé ces résultats.

La plupart des clientes ont déclaré faire grandement confiance à leurs prestataires, bien que les données qualitatives étaient légèrement variables.

Que ce soit en début ou en fin d'intervention, la quasi-totalité des clientes ont affirmé faire confiance à leur prestataire et avoir le sentiment qu'il se souciait de leur bien-être et de leur satisfaction. De même, la quasi-totalité des prestataires ont affirmé que la satisfaction de leurs clientes était importante ou très importante pour eux. Cependant, les réflexions des clientes et les résultats qualitatifs découlant d'autres questions quantitatives étaient mitigés, comme décrit dans la section suivante. Par exemple, plusieurs clientes ont déclaré avoir éprouvé un sentiment de soulagement lorsqu'on leur a promis les soins attentionnés décrits dans la promesse prestataire-cliente, ce qui porte à croire que certaines clientes doutent du traitement qu'elles recevraient sans cette promesse.
Les clientes ont déclaré être convaincues que le prestataire tiendrait sa promesse, et aucune n'a eu l'impression que la promesse n'avait pas été tenue pendant son accouchement. Les clientes ont également souligné que la promesse leur avait appris quelque chose et qu'elles appréciaient qu'on les consulte et qu'on les implique. Ces remarques laissent à penser que même si les clientes déclarent déjà faire confiance à leurs prestataires dans la plupart des cas, elles trouvent aussi la promesse utile, car elle les rassure quant aux soins qu'elles doivent recevoir.

\section{Attentes et satisfaction des clientes}

Bien que les clientes aient déclaré être satisfaites des soins reçus, leurs attentes en matière de soins respectueux étaient modestes et n'ont pas évolué au cours de la mise en œuvre.

En début d'intervention, à la fois dans les établissements participants et témoins, près de la moitié des clientes ont déclaré s'attendre à ce qu'un prestataire leur crie dessus ou les réprimande, tandis qu'un tiers d'entre elles ont déclaré s'attendre à des insultes, des intimidations, des menaces ou des méthodes coercitives. Plusieurs femmes ont clairement mentionné qu'elles s'attendaient à se faire crier dessus ou gifler parce qu'elles arrivaient en retard à l'établissement, ou simplement parce que d'autres femmes leur avaient dit qu'il en était ainsi.

Même si les clientes s'attendaient à des soins irrespectueux, dans les établissements participants et témoins, la quasi-totalité d'entre elles a aussi déclaré s'attendre malgré tout à ce que les prestataires prodiguent des soins de qualité. Pour les clientes, il semblerait donc que le respect n'entre pas en ligne de compte dans la qualité des soins sous un point de vue purement médical. Le fait de ne pas se faire crier dessus ou de ne pas se faire battre, et l'accompagnement du prestataire dans les activités non essentielles à un bon déroulement de l'accouchement (comme aider à nettoyer le sang après l'accouchement) constituaient des sources de profonde satisfaction par rapport aux soins reçus, plutôt que les exemples de traitements que la cliente est censée recevoir. Nous n'avons constaté aucune différence en termes d'attentes en fin d'intervention.

Le risque de perdre le bébé comptait parmi les préoccupations principales de nombreuses femmes et représentait sans doute le facteur clé de satisfaction par rapport à l'accouchement.

Les données qualitatives recueillies dans les établissements participants et témoins suggèrent que si les femmes ont des attentes modestes pour ce qui est de la qualité interpersonnelle des soins reçus, c'est peut-être dû à leur préoccupation pour la survie du bébé. Plusieurs femmes ont expliqué qu'elles estimaient que le risque de perdre le bébé était tellement grand qu'elles s'interdisaient de nouer des liens affectifs avec lui avant d'avoir la certitude qu'il survive. 
Les clientes ont également signalé que ce qui les préoccupait le plus était de mettre au monde un bébé en bonne santé, et nos données qualitatives suggèrent que même quand les clientes s'attendaient à subir un traitement irrespectueux et de violence, elles ont déclaré être satisfaites des soins reçus, car leur bébé était en bonne santé.

Les boîtes à retours ont révélé une opinion favorable de la part des clientes, et les prestataires ont déclaré qu'ils trouvaient les boîtes utiles pour évaluer la satisfaction des clientes.

Bien qu'elles n'aient eu aucun impact mesurable sur la satisfaction des clientes ou son importance aux yeux des prestataires, les données qualitatives révèlent que les clientes et les prestataires des établissements participants s'accordent à penser, que les boîtes à retours sont utiles. Les prestataires ont décrit la boîte à retours comme un moyen d'évaluer la satisfaction des clientes. L'un d'entre eux a dit :

\begin{abstract}
Nous avons par exemple des clientes insatisfaites qui nous aident à analyser la situation afin de mettre le problème en évidence. Si les clientes sont très satisfaites et que nous avons beaucoup de jetons, cela veut dire que nous faisons bien notre travail et que les clientes apprécient...s'il y a une ou deux choses qui ne leur plaisent pas, nous essayons d'en parler entre nous et de résoudre le problème afin que toutes les mères puissent rentrer chez elles satisfaites.0ui, les professionnels de la santé se donnent beaucoup de mal pour satisfaire au mieux toutes les mères. Nous ne voulons pas avoir à la fois des mères satisfaites et des mères insatisfaites.
\end{abstract}

-Prestataire d'un établissement participant

Les clientes des établissements participants se sont senties valorisées lorsqu'on leur a demandé de faire part de leur niveau de satisfaction dans la boîte à retours. De plus, les clientes ont souvent déclaré que les remarques positives motiveraient les prestataires, tandis que les remarques négatives les encourageraient à changer, ce qui suggère qu'elles croient en l'efficacité du mécanisme de retours.

\section{Nécessité de mener des recherches plus approfondies}

Les résultats de cette étude à petite échelle démontrent sa faisabilité, indiquent que la stratégie d'intervention peut être mise en œuvre efficacement et apportent des éléments de preuve préliminaires quant à son potentiel d'impact. Ces résultats soulignent la nécessité de mener une évaluation à plus grande échelle afin de confirmer l'efficacité des solutions et d'identifier les effets relatifs des cinq composantes de la stratégie d'intervention. Même si les fonds Fresh Start n'étaient pas forcément nécessaires pour convaincre les prestataires qu'ils avaient les moyens de faire évoluer les choses-leur degré de motivation étant en effet déjà élevé en début d'intervention-nous ne les exclurons pas des études futures si le contexte s'y prête.

Bien que ces résultats préliminaires soient prometteurs, l'étude comporte un certain nombre de limitations étant donné sa conception et à sa courte durée. Ces limitations, de même que les résultats prometteurs de l'étude, soulignent la nécessité de mener des recherches plus approfondies. Une étude à plus grande échelle pourrait permettre de tester l'impact de l'intervention de manière plus rigoureuse, de mieux comprendre pourquoi les solutions sont efficaces, et d'apporter les éléments d'information nécessaires pour améliorer en permanence la stratégie d'intervention en vue de renforcer son impact sur la qualité et l'expérience de soins des clientes. De plus, une étude à plus grande échelle pourrait permettre d'appuyer les projets de déploiement futurs en fournissant des informations sur les possibilités d'adaptation des solutions afin de garantir leur impact lors d'un déploiement dans différents contextes.

\section{Promesse en matière d'impact}

Cette évaluation à petite échelle fournit des données probantes prometteuses quant au potentiel des solutions axées sur les comportements pour accroître la prestation de SMR. Bien que nous n'ayons pas été en mesure de distinguer les effets relatifs des cinq composantes de l'ensemble de solutions, nos résultats suggèrent, à une exception près, qu'elles ont contribué de manière significative à ces résultats positifs. Les fonds Fresh Start avaient pour but d'accroître le pouvoir perçu par les prestataires de procéder à des changements positifs pour les clientes et ne semblent pas avoir eu cet effet à Chipata, probablement parce que la perception de ces moyens était déjà élevée parmi cette population de prestataires au cours de l'étude de base. Les quatre autres composantes de l'ensemble de solutions (l'atelier de réflexion, la boîte à outils pour la gestion de la douleur, la promesse prestataire-client et la boîte à retours) semblent toutes avoir contribué aux résultats positifs.

Par ailleurs, comme nous l'avons décrit ici et dans notre dossier sur la mise en œuvre, les prestataires ont réussi à instaurer l'ensemble de solutions, et aussi bien les prestataires que les clientes l'ont considéré comme un outil précieux permettant d'améliorer leur travail ou l'expérience en matière d'accouchement. Nous sommes encouragés par les résultats et, avec SM360+, nous solliciterons des responsables de I'OSD de Chipata et le Ministère de la Santé pour déterminer comment ces résultats prometteurs pourraient être exploités pour orienter les programmes de SMR.

Ces résultats précoces démontrent que des solutions axées sur les comportements peuvent améliorer la prestation de SMR, la qualité des soins et la satisfaction des clientes. Dans 
d'autres cadres où les prestataires sont confrontés à des obstacles en matière de SMR similaires à ceux identifiés dans notre recherche formative, l'adaptation de cet ensemble de solutions pourrait aboutir à des résultats positifs similaires. Les responsables programmatiques intéressés par l'adaptation et la réplication de l'ensemble de solutions sont invités à contacter l'équipe de recherche.

\section{References}

${ }^{1}$ Bibliothèque en santé génésique de l'OMS. 2018. "WHO recommendation on respectful maternity care, » Bibliothèque en santé génésique de l'OMS. Genève : Organisation mondiale de la Santé.

${ }^{2}$ Bohren, Meghan A. et al. 2019. " How women are treated during facility-based childbirth in four countries: a cross-sectional study with labour observations and community-based surveys, » The Lancet 394(10210): 1750-63.

${ }^{3}$ Kruk, Margaret E. et al. 2018. « Disrespectful and abusive treatment during facility delivery in Tanzania: a facility and community survey, " Health Policy and Planning 33(1): e26-33.

\section{Remerciements}

Ce dossier de recherche décrit le travail dirigé par ideas 42 dans le cadre du projet Breakthrough RESEARCH. Ce dossier et le travail qu'il décrit sont rendus possibles grâce aux efforts et au soutien de SafeMotherhood360+, Population Council Zambia et Population Council, Washington, DC.

\section{Citation suggérée :}

Breakthrough RESEARCH. 2020. "Prestation de soins de maternels respectueux : conclusions d'une évaluation à petite échelle à Chipata en Zambie, » Dossier de recherche programmatique. Washington, DC : Population Council.

Crédits photographiques pour la couverture : ideas 42

C2020 The Population Council. Tous droits réservés
Courriel

BreakthroughResearch@popcouncil.org

\section{Breakthrough RESEARCH | Population Council}

4301 Connecticut Ave., NW, Suite 280 | Washington, DC 20008

+12022379400 | breakthroughactionandresearch.org

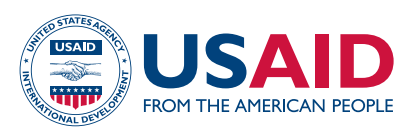

Breakthrough RESEARCH existe grâce au soutien généreux du peuple américain par l'intermédiaire de l'Agence des États-Unis pour le développement international (USAID) aux termes de I'accord de coopération NAID-OAA-A-17-00018. Le contenu de ce document relève de la responsabilité exclusive de Breakthrough RESEARCH et du Population Council et ne reflète pas nécessairement l'opinion de l'USAID ou celle du gouvernement des États-Unis.

\section{POPULATION} COUNCIL

Ideas. Evidence. Impact.

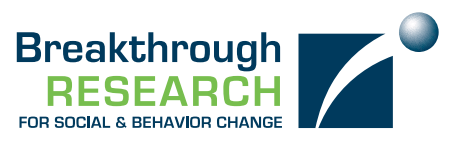

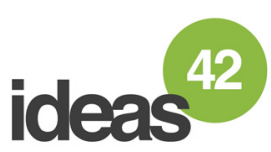

Breakthrough RESEARCH impulse des changements sociaux et comportementaux (CSC) en réalisant des études et des évaluations inédites et en promouvant les solutions fondées sur des données probantes afin d'améliorer les programmes de santé et de développement à travers le monde. Breakthrough RESEARCH est un consortium dirigé par le Population Council, en partenariat avec Avenir Health, ideas42, I'Institute for Reproductive Health de Georgetown University, le Population Reference Bureau et Tulane University. 